\title{
Neutron Correlations with Electrical Measurements in a Plasma Focus Device
}

\author{
H. Bruzzone ${ }^{1}$, H. Acuña ${ }^{1}$, and A. Clausse ${ }^{2}$ \\ ${ }^{1}$ Universidad de Mar del Plata and CONICET, 7600 Mar del Plata, Argentina \\ ${ }^{2}$ CNEA-CONICET and Universidad Nacional del Centro, 7000 Tandil, Argentina
}

Received on 22 October, 2007

\begin{abstract}
The measurement of the voltage between electrodes and the discharge current time derivative in Plasma Focus devices can be used to obtain important information on the neutron-producing pinch stage of the mentioned devices. The analysis of a 60 -shots experimental run in a $5.7 \mathrm{~kJ}$ Mather-type device results in correlations suggesting that the neutron yield depends mostly on the average energy per particle delivered to the plasma during the pinch stage.
\end{abstract}

Keywords: Plasma Focus; Neutrons; Signal Processing; Plasma Diagnostics

\section{INTRODUCTION}

Plasma Focus (PF) devices are a special type of pulsed, coaxial, microsecond gas discharges which manage to produce plasma conditions such that energetic radiation is emitted ( $\mathrm{X}$ rays, ions and electrons beams and, if filled with Deuterium or Deuterium-Tritium, fusion neutrons). Since their discovery around 1960 by N. V. Filippov (Petrov et al 1960) and J. W. Mather (1965), PF devices have been studied in many laboratories throughout the world, but nevertheless the physical processes underlying its behavior are still a matter of controversy. It is known that an optimal filling pressure value exists for any device at which the neutron yield $Y_{n}$ attains its maximum value, however, there is not yet a conclusive agreement regarding the reasons for the existence of such optimum pressure (the naïve concept of requiring that the pinch occurs at maximum discharge current simply does not hold in most of the experimental situations). Moreover, it is neither understood why the numerous attempts to achieve neutron yields higher than $\approx 10^{12}$ per shot were fruitless. It is known that, in all the devices, $Y_{n}$ values fluctuate on a shot to shot basis under apparently identical operating conditions (electrodes geometry, voltage, pressure); but it is not clear why this is so. Finally, whether the neutrons are produced by a thermal plasma, a beam of high-energy deuterons, or a mix of both, is still a debated question (Zakaullah et. al 2001). Hence, a lot of systematic work on these devices is still needed to reach a proper understanding of their functioning, and further take advantage of their multiple potential applications.

This article reports a technique of acquiring knowledge on plasma properties more closely related to the neutron production than the mere "external" parameters like bank energy or peak discharge current, which can be evaluated from relatively simple measurements of the discharge current and voltage between the electrodes. The technique is applied for analyzing a set of 60 shots performed at 6 different $\mathrm{D}_{2}$ filling pressures in a relatively small PF device at fixed bank energy in an attempt to find some insight into the shot to shot variations and on the pressure dependence of the neutron yield.

\section{DIRECT EMPIRICAL CORRELATIONS}

Bruzzone et al (2006) showed that using measurements of the voltage on the collector plates of a PF device, $V(t)$, and the time derivative of the discharge current, $d I / d t$, the inductance of the plasma-coaxial electrodes system, $L_{p}(t)$, can be determined. Using this technique, $L_{p}$ was determined in a $30 \mathrm{kV}$, $12.6 \mu \mathrm{F}, \mathrm{PF}$ device (inner electrode radius $a=1.8 \mathrm{~cm}$, outer electrode radius $b=3.6 \mathrm{~cm}$, electrodes length $d=10 \mathrm{~cm}$ ) operating at 6 different pressure values $\left(p_{o}=1,2,3,4,5\right.$ and 6 mbar). Plots of the experimental data of $V(t), d I / d t$ and the corresponding $L_{p}(t)$ are shown in Fig. 1 for a shot performed at 2.10 mbar. It can be seen that the pinch stage, recognized by the dip in $d I / d t$ and the spike in $V(t)$, is accompanied by a sudden rise in $L_{p}(t)$. The value of $L_{p}$ just before the jump (later on called $\left.L_{\text {coax }}\right)$ is about $10.5 \mathrm{nH}$, is rather invariant for all the shots and pressures, and matches well with the inductance of the electrodes system closed at its end by a bullet shaped current sheet. The values of the inductance jump associated with the pinch stage, $\Delta L_{p}$, change from shot to shot, but are in reasonable agreement with those expected of a typical pinch column.

The determination of $L_{p}(t)$ from the electrical signals is based on two assumptions, namely, that a single current sheet exists within the electrodes and that no relevant resistance exists in the plasma. Regarding the first assumption, experimental evidence was presented in a few papers sustaining the presence of a secondary current sheet (so called leakage currents). However, it should be also stressed that this is not an universal feature of PF devices. In particular, in the present experiment the existence of leakage currents is not consistent with the fact that the determined values of $L_{p}(t)$ agree fairly well with those expected for a single sheet. On the other hand, the existence of so called anomalous resistances is a controversial issue. Up to now there is no direct measurements of such resistance, the references found in the literature claiming its existence being based mainly on theoretical speculations and indirect experiments. In any case, as discussed in Bruzzone et al (2006), the values of $\Delta L_{p}$ found in the present experiment are consistent with those expected for a typical pinch column, so that there is no reason to consider eventual anomalous resistivities.

Details of the experiment and $L_{p}(t)$ evaluation can be found in Bruzzone et al (2006). At each of the six initial filling pres- 


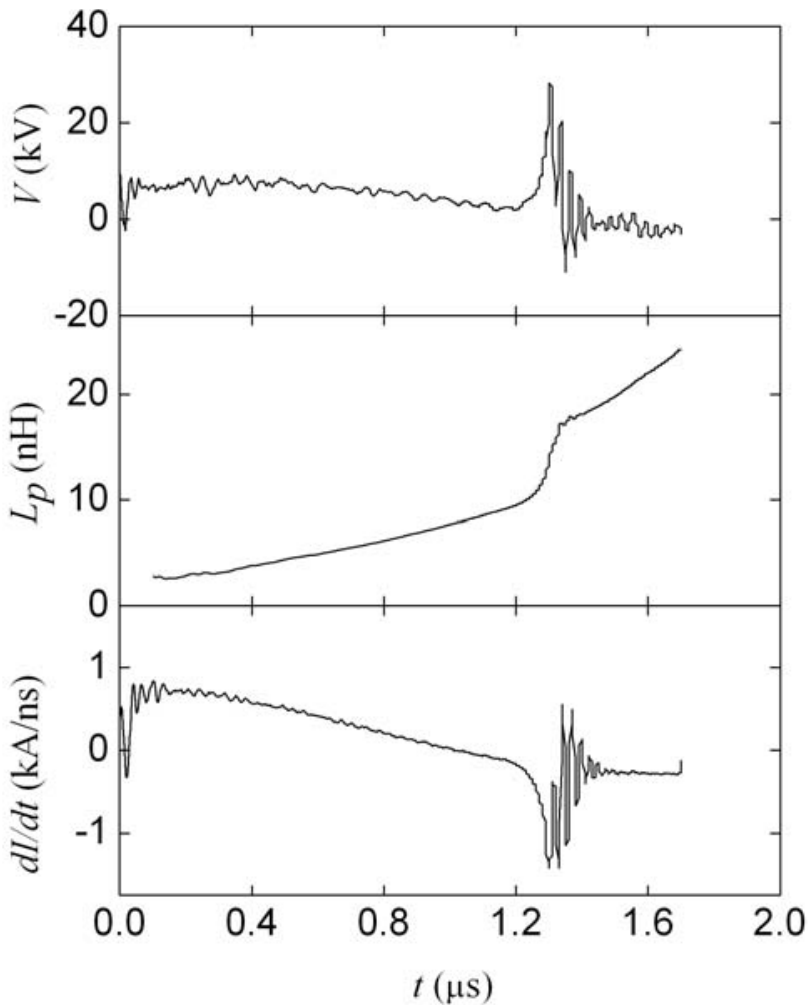

FIG. 1: Voltage on the collector plates, $V(t)$, time derivative of the discharge current, $d I / d t$, and inductance of the plasma-coaxial electrodes system, $L_{p}(t)$, operating at 2.10 mbar.

sure values mentioned above, ten consecutive shots were performed before evacuating the vacuum chamber. The experiment was performed using a fore-diffusion vacuum pumps system, which provided a base vacuum well below $10^{-5}$ mbar. After several days of pumping, the chamber leakage was controlled and found well below the 0.1 mbar range after several hours (a time interval smaller that that required to make 10 shots). The chamber was previously conditioned by numerous preliminary shots performed in Deuterium without breaking the vacuum, in order to ensure that the adsorbed gas in the chamber walls and electrodes was essentially Deuterium. The pressure variations found after each shot were "jumps" natured (the rise in pressure, if any, takes place immediately after the shot without further changes), as expected from outgassing due to plasma-wall interactions. Furthermore, the assumption that Deuterium is the main component of the pressure increase is supported by the fact that in every series the largest neutron yield never occurred at the first shot (i.e.the one performed on the purest gas) but about the middle of the series, which would have not been the case if the pressure increased due to contamination with other gases. In any case, we want to stress the fact that the fluctuation in the neutron yield on a shot to shot basis at fixed pressure values is a standard PF devices behavior even changing the gas every shot.

Table 1 presents the values of $p_{o}, Y_{n}, \Delta L_{p}$ and the current $I_{p}$ at the start of the pinch formation, for each shot. Absence of data means that for this shot, neither dip in $d I / d t$, nor peak in $V(t)$ nor neutrons were observed. Plots of the pressure dependence of the average $\left\langle Y_{n}\right\rangle$ and the maximum $Y_{n}^{\max }$ neutron yields are given in Fig. 2. It can be seen that both present a maximum at 3 mbar in this particular device.

Bernard et al (1977) found that the average neutron yield correlates with the average pinch current as $I_{p}^{3.3}$. This correlation was obtained using data from several different devices operating in their optimized configuration (that is, that yielding the highest neutron yield). It is then reasonable to search for a correlation between the values of $Y_{n}$ and $I_{p}$ in every shot. Fig. 3 shows how the individual shot pairs $\left(Y_{n}, I_{p}\right)$ distribute. It can be seen that there is a certain regularity, however the correlation coefficient between both is just 0.25 .

There is a physical reason to correlate $Y_{n}$ with $\Delta L_{p}$, based on the fact that larger pinch-inductance jumps strongly suggest larger pinch lengths (the logarithm dependence on radius is to weak to account for the differences), which would imply either larger thermonuclear reacting-plasma volumes and/or larger paths for deuteron beams. Fig. 4 shows the distribution of the individual shot pairs $\left(Y_{n}, \Delta L_{p}\right)$. It can be seen that the relation between both variables is also weak, and the correlation coefficient between $Y_{n}$ with $\Delta L_{p}$ is barely 0.37 .

\section{ENERGY CONSIDERATIONS}

Better information can be extracted from the measurements by introducing some processing and analysis of the electrical signals. After the coaxial stage the discharge can be described by the equivalent circuit shown in Fig. 5. $L_{0}^{\prime}$ is a fixed external inductance due to the back plate connections, $L_{p}=L_{\text {coax }}+L_{p}^{\prime}(t)$ is the electrodes-plasma inductance, and $L_{p}^{\prime}(t)$ is the additional inductance produced by the collapsing current sheet. The voltage between the electrodes is accordingly given by:

$$
\begin{gathered}
V(t)=\left(L_{o}^{\prime}+L_{\text {coax }}\right) \frac{d I}{d t}+\frac{d}{d t}\left(L_{p}^{\prime} I\right) \\
=\left(L_{o}^{\prime}+L_{\text {coax }}\right) \frac{d I}{d t}+V_{p}(t)
\end{gathered}
$$

where $V_{p}(t)$ is the voltage drop on the collapsing current sheet and subsequent pinch. Therefore, $V_{p}$ can be determined from the measured values of $V(t), d I / d t, L_{o}^{\prime}(=6.2 \pm 0.2 \mathrm{nH})$ and $L_{\text {coax }}$. Furthermore, multiplying $V(t)$ by $I(t)$ and integrating between the end of the coaxial run, $t_{c}$, and any time $t_{i} t_{c}$ one obtains the corresponding energy delivered to this portion of the circuit, $\Delta E(t)$ as

$$
\Delta E(t)=\frac{1}{2}\left(L_{o}^{\prime}+L_{\text {coax }}\right)\left[I^{2}(t)-I^{2}\left(t_{c}\right)\right]+\int_{t_{c}}^{t} I d\left(I L_{p}^{\prime}\right)
$$

The first term is the magnetic-energy variation in the fixed inductances, $\Delta E_{m}$, whereas noting that $L_{p}^{\prime}\left(t_{c}\right)$ is null by definition, the second term can be integrated twice by parts leading to: 
TABLE 1. Experimental log (Absence of data means that for this shot, neither dip in $d I / d t$, nor peak in $V(t)$ nor neutrons were observed).

\begin{tabular}{|c|c|c|c|c|c|c|}
\hline \# Shot & $p_{o}(\mathrm{mbar})$ & $Y_{n}\left(10^{6}\right)$ & $\Delta L_{p}(\mathrm{nH})$ & $I_{p}(\mathrm{kA})$ & $V_{p}^{\max }(\mathbf{k V})$ & $\Delta E_{p}^{\max }(\mathbf{J})$ \\
\hline 1 & 1.00 & -- & ----- & --- & --- & --- \\
\hline 2 & 1.10 & 28 & 5.8 & 433 & 51 & 237 \\
\hline 3 & 1.15 & -- & ----- & --- & --- & --- \\
\hline 4 & 1.20 & 31 & 5.7 & 441 & 32 & 194 \\
\hline 5 & 1.20 & -- & ----- & --- & --- & --- \\
\hline 6 & 1.20 & 12 & 4.7 & 440 & 27 & 207 \\
\hline 7 & 1.20 & -- & ----- & --- & --- & --- \\
\hline 8 & 1.20 & 27 & 11.8 & 440 & 44 & 410 \\
\hline 9 & 1.25 & 1 & ----- & --- & --- & --- \\
\hline 10 & 1.30 & 34 & 9 & 439 & 51 & 335 \\
\hline 1 & 2.00 & -- & ----- & --- & --- & --- \\
\hline 2 & 2.00 & 33 & 3.1 & 445 & 38 & 216 \\
\hline 3 & 2.00 & 8 & 3.4 & 449 & 26 & 144 \\
\hline 4 & 2.00 & --- & ----- & --- & --- & --- \\
\hline 5 & 2.00 & 45 & 7.3 & 438 & 49 & 247 \\
\hline 6 & 2.00 & --- & ----- & --- & --- & --- \\
\hline 7 & 2.05 & 21 & 4.1 & 465 & 44 & 138 \\
\hline 8 & 2.05 & --- & ----- & --- & --- & --- \\
\hline 9 & 2.10 & 24 & 8 & 461 & 12 & 314 \\
\hline 10 & 2.10 & 3 & 3 & 449 & 28 & 125 \\
\hline 1 & 3.00 & 12 & 2.8 & 449 & 8.4 & 152 \\
\hline 2 & 3.00 & 17 & 5 & 455 & 28 & 193 \\
\hline 3 & 3.00 & 86 & 7.3 & 460 & 50 & 290 \\
\hline 4 & 3.00 & 118 & 7.4 & 451 & 47 & 276 \\
\hline 5 & 3.10 & 40 & 6.4 & 456 & 57 & 278 \\
\hline 6 & 3.10 & 21 & 4.1 & 462 & 41 & 207 \\
\hline 7 & 3.10 & 67 & 9 & 458 & 55 & 310 \\
\hline 8 & 3.10 & 78 & 9 & 458 & 64 & 305 \\
\hline 9 & 3.20 & 7 & 3.5 & 454 & 23 & 138 \\
\hline 10 & 3.20 & 25 & 7.6 & 445 & 53 & 256 \\
\hline 1 & 4.00 & 1 & 4 & 453 & 14 & 133 \\
\hline 2 & 4.10 & 14 & 6.9 & 464 & 34 & 246 \\
\hline 3 & 4.10 & 22 & 6.8 & 456 & 43 & 238 \\
\hline 4 & 4.15 & 44 & 7.8 & 451 & 41 & 231 \\
\hline 5 & 4.15 & 21 & 5.4 & 468 & 46 & 250 \\
\hline 6 & 4.20 & 13 & 7.5 & 461 & 41 & 252 \\
\hline 7 & 4.20 & 30 & 6.9 & 459 & 44 & 238 \\
\hline 8 & 4.20 & 5 & 7.2 & 467 & 35 & 233 \\
\hline 9 & 4.20 & 7 & 7.5 & 446 & 43 & 192 \\
\hline 10 & 4.25 & 30 & 8.5 & 471 & 42 & 276 \\
\hline 1 & 5.00 & $\begin{array}{l}--- \\
\end{array}$ & ----- & --- & --- & --- \\
\hline 2 & 5.10 & 23 & 7 & 466 & 5 & 208 \\
\hline 3 & 5.10 & 9 & 7.7 & 456 & 40 & 196 \\
\hline 4 & 5.10 & 28 & 8.7 & 470 & 26 & 220 \\
\hline 5 & 5.20 & 5 & 7.5 & 453 & 33 & 180 \\
\hline 6 & 5.20 & 6 & 6.2 & 458 & 27 & 172 \\
\hline 7 & 5.20 & 6 & 7.5 & 452 & 40 & 176 \\
\hline 8 & 5.20 & 14 & 6.2 & 461 & 25 & 174 \\
\hline 9 & 5.20 & 8 & 8 & 456 & 34 & 172 \\
\hline 10 & 5.20 & 5 & 6.2 & 456 & 34 & 169 \\
\hline 1 & 6.00 & --- & ----- & --- & --- & --- \\
\hline 2 & 6.05 & 2 & 5.7 & 460 & 24 & 128 \\
\hline 3 & 6.10 & 2 & 5.4 & 467 & 25 & 126 \\
\hline 4 & 6.10 & 4 & 6.2 & 474 & 37 & 185 \\
\hline 5 & 6.15 & 1 & 3.2 & 455 & 11 & 75 \\
\hline 6 & 6.20 & 2 & 6.6 & 462 & 31 & 162 \\
\hline 7 & 6.20 & 4 & 6.7 & 473 & 24 & 176 \\
\hline 8 & 6.20 & 5 & 6.4 & 461 & 34 & 176 \\
\hline 9 & 6.20 & 2 & 6.5 & 468 & 27 & 125 \\
\hline 10 & 6.30 & 2 & 4.2 & 457 & 11 & 90 \\
\hline
\end{tabular}




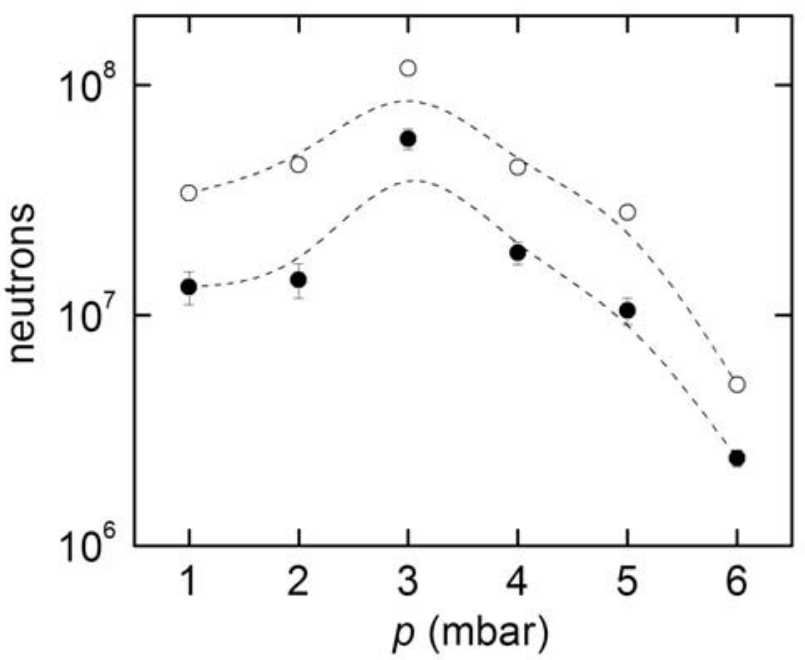

FIG. 2: Dependence of the average and maximum measured neutron yield on the filling $\mathrm{D}_{2}$ pressure.

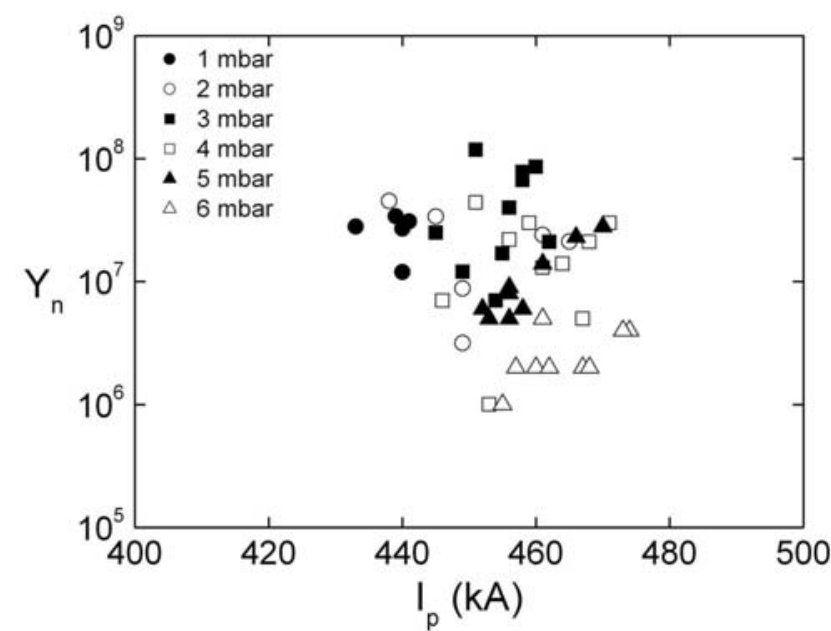

FIG. 3: Neutron yield and current value at the start of the pinch formation in each neutronic shot.

$$
\Delta E(t)=\Delta E_{m}+\frac{1}{2} L_{p}^{\prime} I^{2}(t)+\frac{1}{2} \int_{t_{c}}^{t} I^{2} d L_{p}^{\prime}
$$

The second and third term are the magnetic-energy variation in the plasma variable inductance and the electromechanical work done on the pinch (Bruzzone et al (1976)), $\Delta E_{p}$, that is:

$$
\Delta E_{p}(t)=\frac{1}{2} \int_{t_{c}}^{t} I^{2} d L_{p}^{\prime}
$$

Figure 6 shows, as examples, $V_{p}(t)$ and $\Delta E_{p}(t)$ evaluated for shots at 2.10 and 6.10 mbar. Values of $V_{p}$ and $\Delta E_{p}$ were

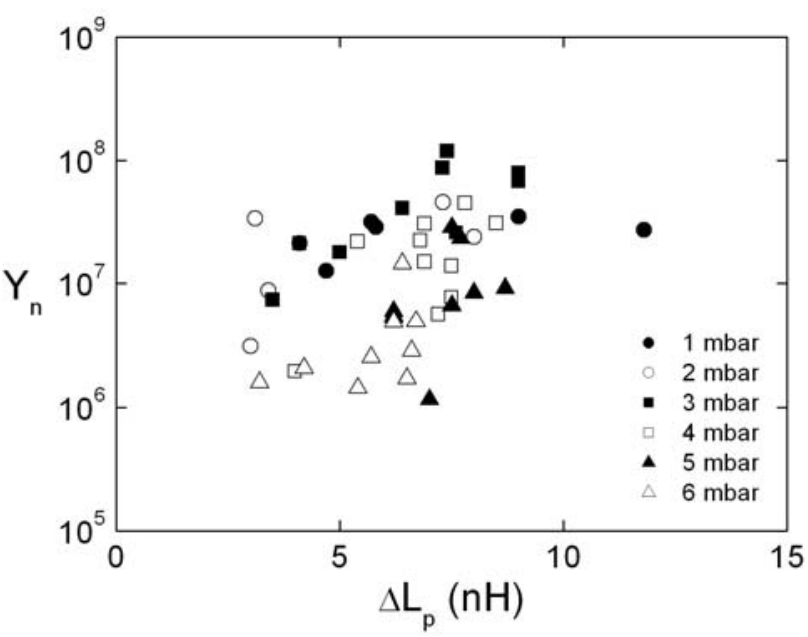

FIG. 4: Neutron yield and pinch-inductance jump in each neutronic shot.

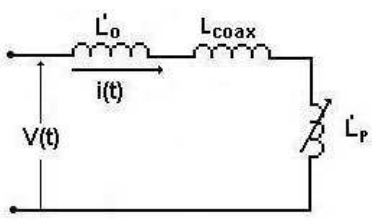

FIG. 5: Equivalent circuit after the end of the coaxial stage.

calculated for each shot displaying both a dip in $d I / d t$ and a measurable neutron yield. A standard pattern was found: $V_{p}$ goes through a maximum value, and $\Delta E_{p}$ increases monotonically during the pinch phase up to a value $\Delta E_{p}^{\max }$, which is defined as the value of $\Delta E_{p}$ at the time the $L_{p}$ jump ends (which coincides with the end of the peaks in $V(t)$ and $d I / d t)$. It is important to note that the values of $V_{p}$ experimentally determined using this procedure are a sound measurement of the voltage drop on the pinch column, independently of the physical interpretation of the nature of such drop. In fact, even if the physical cause of the dip in $d I / d t$ should be assigned to a resistive phenomena instead of an inductive one (i.e., that the last term in the right hand side of Eq.(1) could be alternatively written as a resistance times the current instead of the time derivative of a magnetic flux) the evaluation of $V_{p}$ is just the same. Hence, the values of pinch voltages presented in this work are not theory dependent.

It is reasonable to explore a correlation between and the neutron yield, since the pinch voltage can be related to the energy and intensity of eventual deuteron beams. On the other hand, $\Delta E_{p}^{\max }$, is the thermal and eventually kinetic plasma flow energy delivered to the particles trapped inside the pinch, related to thermonuclear yields. In Table 1 , the values of and $\Delta E_{p}^{\max }$ are given for each neutronic shot. Plots of $Y_{n}$ vs $V_{p}^{\max }$ and $Y_{n}$ vs $\Delta E_{p}^{\max }$ are given in the Figures 7 and 8 , respectively. Using these parameters, the correlation coefficients improve: they are 0.66 in the case of $V_{p}^{\max }$ and 0.63 in the case of $\Delta E_{p}^{\max }$.

Models of thermonuclear and beam-target neutron production in pinch columns have been developed, linking the ex- 

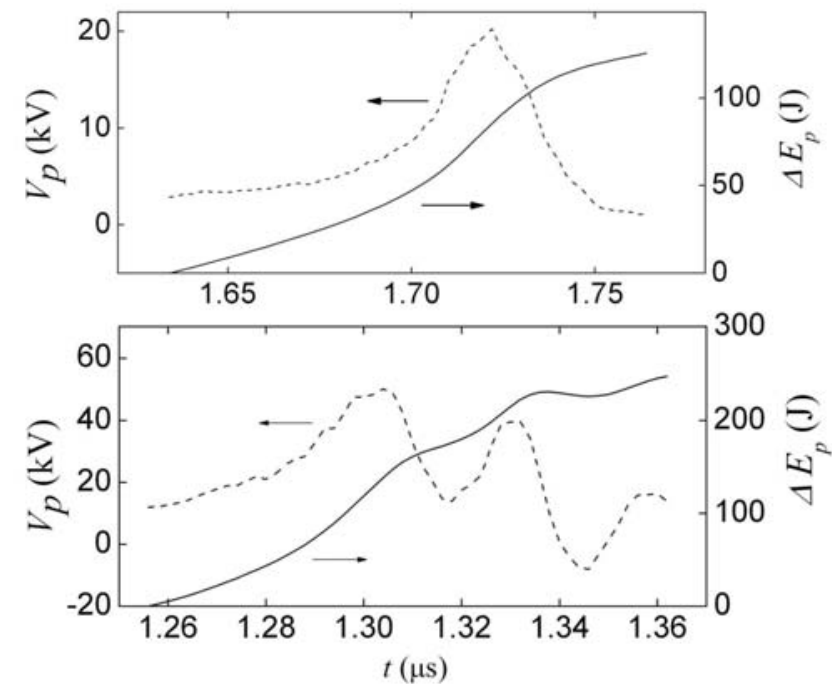

FIG. 6: Voltage between electrodes and electromechanical work over the pinch (upper $6.10 \mathrm{mbar}$, lower $2.10 \mathrm{mbar}$ ).

pected yield to other plasma parameters. Using them (see Appendix 1) one can obtain possible dependences of the yield in both cases. In the thermonuclear scenario, a dependence of $Y_{n} / p_{o}^{2}$ with $\varepsilon=\Delta E_{p}^{\max } / p_{o}$ is expected, while in a simple beamtarget one a dependence of $Y_{n} / p_{o}$ with $V_{p}^{\max }$ should hold. The details are given in the Appendix 1, and using the data presented in this work, the correlation coefficient for the thermonuclear case is 0.79 while that of the simple beam target case is 0.30 .

\section{CONCLUSIONS}

Using standard voltage and discharge-current time derivative measurements, it is possible to determine, in any PF shot, the CS-electrodes system inductance and from it, in neutron producing shots, relevant physical magnitudes like $\Delta L_{p}, V_{p}^{\max }$ and $\Delta E_{p}^{\text {pinch }} / p_{o}$. The determination of the variable inductance of a PF discharge from simple voltage and discharge current time derivative measurements allows them for a deeper insight of the device behavior in any shot, and becomes an interesting diagnostic technique for the study of these devices.

Comparisons between these parameters and the neutron production in a systematic 60-shots experimental run on a small PF device yielded poor correlations with $I_{p}$ and $\Delta L_{p}$. Hence, neither the shot to shot fluctuations at fixed pressure nor the variations in yield with the pressure can be explained in terms of these parameters. A somewhat better correlation of the neutron yield with $V_{p}^{\max }$ and $\Delta E_{p}^{\max }$ was found, suggesting that these parameters are more directly related to the neutron production. The best correlation found was with $\varepsilon$, proportional to the energy delivered per particle during the pinch stage. Due to the fact that $\varepsilon$ can be reasonably assumed to be proportional to the average ion temperature in the pinch, the data set analyzed in this work is more consistent with a ther- monuclear scenario than with a beam-target picture. However, the beam-target model presented in this article is just a possible scenario, and other more complex models have been proposed (Jäger and Herold 1987). Anyhow, it should be stressed that although the present diagnostic introduces new and interesting information, the results have not enough breadth to allow for any general conclusion on the neutron generation mechanism.

The patterns found and reported in this paper can be useful to guide future efforts toward the complete understanding of the physical mechanisms involved in the radiation emissions from Plasma Focus discharges. It is in fact surprising that a relative small data set can reveal interesting dependences among physically meaningful magnitudes. These results are encouraging in the sense that future experimental studies can unveil more interesting correlations.

\section{APPENDIX 1}

\section{Thermonuclear neutron yield per pair of particles}

Assuming that neutrons are produced by thermonuclear reactions in the pinch, the neutron yield is given by [Clausse $e t$ al 1998]:

$$
Y=\frac{1}{2} \int_{\Delta t} n^{2}<\sigma v>V d t
$$

where $n$ is the deuteron density in the pinch, $\langle\sigma v\rangle$ is the thermal cross section, and $V$ and $\Delta t$ are the volume and duration of the pinch. Moreno et al [2000] have shown that under certain assumptions about the averages, Eq. (A1) leads to:

$$
\frac{Y}{N^{2} / 2}=\frac{<\sigma v>_{B} \Delta t}{V_{B}} \propto T_{B}^{5 / 12} \exp \left[-\left(\frac{T_{B}}{T^{*}}\right)^{-1 / 3}\right]
$$

where $N$ is the number of deuterons in the pinch, the subindex $B$ stands for the Bennett condition, $T$ is the temperature, and $T^{*}$ is a constant parameter of the Deuterons fusion cross section (a "temperature" corresponding to $6.6 \mathrm{MeV}$ ).

Assuming that $N$ is proportional to $p_{o}$ (the number of particles entailed into the pinch should increase linearly with the neutral filling density), the magnitude $\varepsilon=\Delta E_{p}^{\max } / p_{o}$ should be proportional to the energy per particle which, if the kinetic energy of plasma movements can be disregarded, is actually the temperature. One can then explore the correlation of $Y_{n} / p_{o}^{2}$ (which is proportional to the yield per particle pair) with $\varepsilon$. Fig. A1 shows the experimental points depicted in the plane $\left(\frac{Y_{n}}{p_{o}^{2}}, \varepsilon\right)$. The curve appearing in the graphic corresponds to the best fit of the function:

$$
\frac{Y_{n}}{p_{o}^{2}}=A \varepsilon^{5 / 12} \exp \left[-\left(\frac{\varepsilon}{\varepsilon_{o}}\right)^{-1 / 3}\right]
$$

which matches Eq. A2 provided that $\varepsilon$ is proportional to the Bennett temperature. The correlation factor of the fitting is 


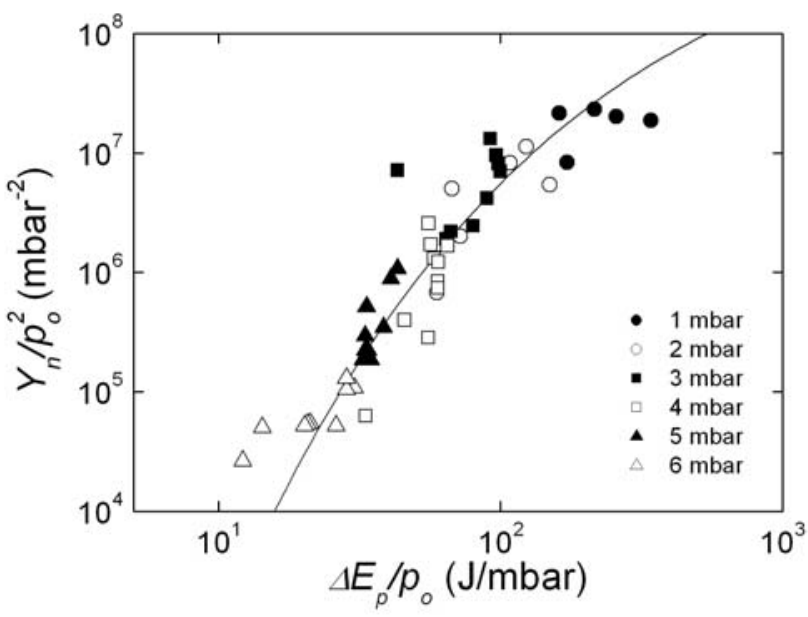

FIG.1A. Correlation between the neutron yield per particle pair and the energy per particle delivered to the pinch.

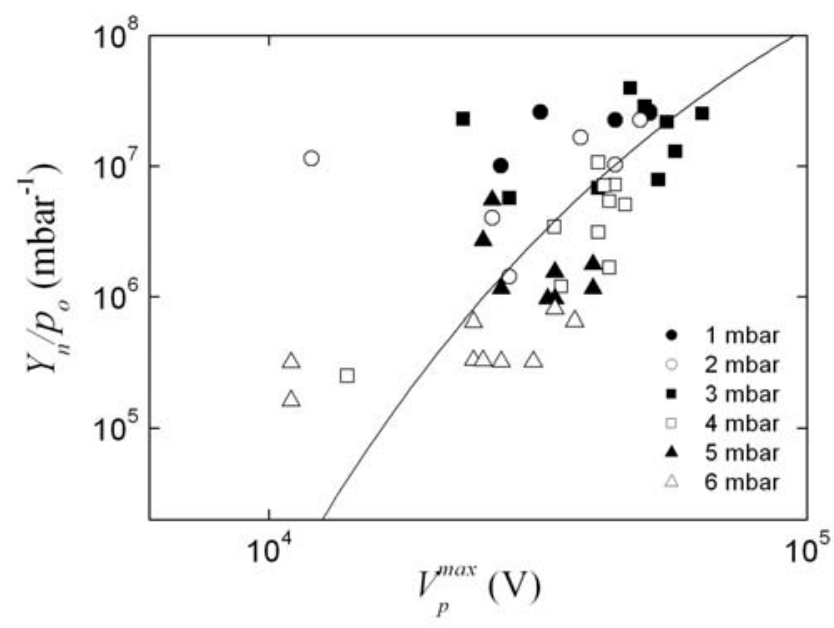

FIG.2A. Correlation between the neutron yield per particle and the maximum pinch voltage.

0.79. The best estimates of the constant parameters of the formula are:

$$
\begin{aligned}
& A=1.0710^{11} \mathrm{mbar}^{-2}(\mathrm{~J} / \mathrm{mbar})^{-5 / 12} \\
& \varepsilon_{o}=58000 \mathrm{~J} / \mathrm{mbar}
\end{aligned}
$$

which in principle should be related to physical properties and to specific features of the device.

\section{Beam-target neutron yield per particle}

Assuming that neutrons are produced by beam-target reactions in the pinch, the neutron yield should go as:

$$
Y_{b t} \propto N_{i} \lambda n_{o}
$$

where $N_{i}$ is the number of accelerated ions, $n_{o}$ is the density in the plasma (i.e.the average pinch density), and $\lambda$ is the mean free path of an ion. The latter is proportional to $1 / n_{o}$ [Huba 2006]. Assuming that $N_{i}$ is proportional to $n_{o}$ and that $n_{o}$ is proportional to the filling pressure $p_{o}$, it can be concluded that $Y_{b t} \propto p_{o}$.

Figure A2 shows the distribution of the experimental points on the plane $\left(Y / p_{o}, V_{p}^{\max }\right)$. It can be seen that the visual correlation is much weaker than that in Fig. A1. $Y_{b t}$ follows an exponential dependence respect to the accelerating voltage, $V$, that is [McCall 1989]:

$$
\frac{Y_{b t}}{p_{o}} \propto \lambda v_{i} V^{-1} \exp \left(-\frac{A_{1}}{V^{0.5}}\right) \propto \exp \left(-\frac{A_{1}}{V^{0.5}}\right)
$$

where $v_{i}$ is the velocity of the accelerated ions - - proportional to $\sqrt{V}--, \lambda$ is the mean free path -- proportional to $v_{i}--$, and $A_{1}$ equals 1500 in mks units.

The curve shown in Fig. 8 corresponds to the best fit of Eq. A5, that is:

$$
\frac{Y_{n}}{p_{o}}=1.310^{10} \mathrm{mbar}^{-1} \exp \left(-\frac{A_{1}}{V^{0.5}}\right)
$$

The corresponding correlation factor is 0.30 .
[1] A. Bernard, P. Cloth, H. Conrads, A. Coudeville, C. Gourlan, A. Jolas, C. Maisonnier, J.P. Rager, Nucl. Instr. and Meth. 145, 191 (1977).

[2] A. Bernard, H. Bruzzone, P. Choi, H. Chuaqui, V. Gribkov, J. Herrera, K. Hirano, A. Krejci, S. Lee, C. Luo, F. Mezzetti, M. Sadowski, H. Schmidt, K. Ware, C. S. Wong, and V. Zoita, J. Moscow Phys. Soc. 8, 93 (1998)

[3] H. Bruzzone, H. Kelly, M. Milanese, and J. Pouzo, Nuclear Fusion 16, 870 (1976).

[4] H. Bruzzone, H. Acuña, M. Barbaglia, and A. Clausse, Plasma Phys. and Controll. Fusion, 48, 609 (2006).

[5] A. Clausse, L. Bilbao, and H. Bruzzone, Journal of Technical Physics, 39, 33 (1998).

[6] J. D. Huba, (2006) revised NRL plasma Formulary, Naval Re- search Laboratory, Washington.

[7] V. Jäger and H. Herold, Nuclear Fusion 27, 407 (1987).

[8] J. W. Mather, Phys. Fluids, 8, 366 (1965).

[9] G. McCall, Phys. Rev. Lett. 62, 1986 (1989).

[10] C. Moreno, H. Bruzzone, J. Martínez, and A. Clausse, IEEE Transactions on Plasma Science, 28, 1735 (2000).

[11] D. Petrov, N. Filippov, T. Filippova, and V. Khrabrov, (1960) Powerful pulsed gas discharges in chambers with conducting walls, in Plasma Physics and the problem of Controlled Thermonuclear Reactions, IV p. 198-212.

[12] M. Zakaullah, K. Alamgir, A. Rasool, M. Shafiq, M. Murtaza, and A. Waheed, IEEE Transactions Plasma Science, 29, 62 2001). 